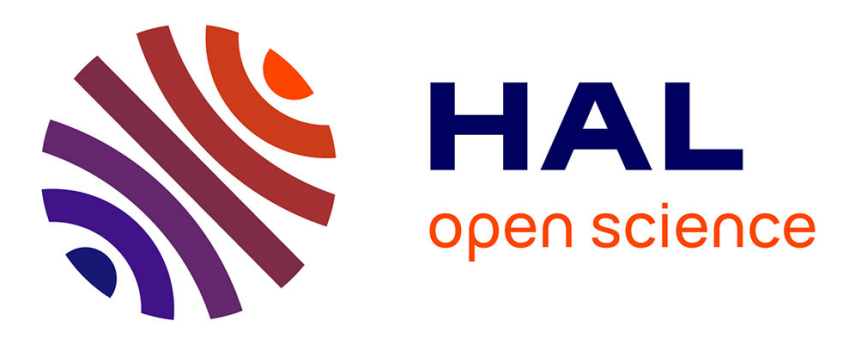

\title{
Topology of dynamical reconstructions from Lagrangian data
}

\author{
Gisela Charó, Guillermo Artana, Denisse Sciamarella
}

\section{To cite this version:}

Gisela Charó, Guillermo Artana, Denisse Sciamarella. Topology of dynamical reconstructions from Lagrangian data. Physica D: Nonlinear Phenomena, 2020, 405, pp.132371. 10.1016/j.physd.2020.132371 . hal-03036018

\section{HAL Id: hal-03036018 https://hal.science/hal-03036018}

Submitted on 2 Dec 2020

HAL is a multi-disciplinary open access archive for the deposit and dissemination of scientific research documents, whether they are published or not. The documents may come from teaching and research institutions in France or abroad, or from public or private research centers.
L'archive ouverte pluridisciplinaire HAL, est destinée au dépôt et à la diffusion de documents scientifiques de niveau recherche, publiés ou non, émanant des établissements d'enseignement et de recherche français ou étrangers, des laboratoires publics ou privés. 


\title{
Topology of dynamical reconstructions from Lagrangian data
}

\author{
Gisela D. Charó ${ }^{\mathrm{a}, \mathrm{b}}$, Guillermo Artana ${ }^{\mathrm{a}, \mathrm{b}}$, Denisse Sciamarella ${ }^{\mathrm{c}}$ \\ ${ }^{a}$ Laboratorio de Fluidodinámica, Facultad de Ingeniería, Universidad de Buenos Aires, \\ C1063ACV CABA, Argentina \\ ${ }^{b}$ CONICET - Consejo Nacional de Investigaciones Científicas y Técnicas, C1425FQD CABA, \\ Argentina \\ ${ }^{c}$ Institut Franco-Argentin d'Études sur le Climat et ses Impacts (IFAECI), UMI 3351 \\ (CNRS-CONICET-UBA), C1428EGA CABA, Argentina
}

\begin{abstract}
Branched Manifold analysis through Homologies (BraMAH) is a technique that computes the state-space topology of a dynamical reconstruction from scalar data. This work introduces the application of this technique to Lagrangian time series. The approach unveils the topological structure underlying the behavior of a fluid particle. When applied to a set of sparse particles, the results of the analysis can be used to classify them according to the dynamics they deploy during a given time window. Topological grids can be constructed to portray the spatial organization of the topological classes. The connection between the topological grids and the transport properties of the flow is examined using streaklines. Even if demonstrated here in the context of kinematic flow models, the generality of the method allows for its potential application to experimental or observational Lagrangian data satisfying the technical requirements for the analysis.
\end{abstract}

Keywords: Topology, Delay-coordinate embedding, Nonlinear time-series analysis, Homology

\section{INTRODUCTION}

The interest of topology within nonlinear dynamics dates back to Poincaré, who had already noticed the way in which a dynamical system's properties depend upon topology [1]. With the latest advances in computational topology [2],

Email address: gcharo@fi.uba.ar (Gisela D. Charó) 
the subject is attracting once again the attention of dynamicists who considered applying general pattern recognition concepts $[3,4,5]$ to the spatio-temporal patterns emerging in nonlinear systems involving diffusion and convection mechanisms $[6,7,8,9,10,11]$. In the field of Fluid Dynamics, additional important contributions can be found in [12]. Contemporaneously, scientists started adapting the newly developed topological tools to the state-space reconstruction of dynamical systems $[13,14,15]$. This research line builds upon earlier studies that proposed the extraction of Betti numbers from time series [16]. All these methods rely on the construction of a cell complex, as defined in algebraic topology [17]. In chaos topology [18], the cell complex must be built from a point cloud that is obtained from a time series with embedding techniques [19]. The topology associated to the time series is unveiled computing the homology groups of the constructed cell complex, but the cell complex can be constructed in many nonequivalent ways. Standard computational strategies to compute homologies from point clouds [20, 21, 22] have some points in common: the points of the cloud are vertices of the cells; the cells are simplices; and connections between the vertices of the cells are determined by their nearness. For the algorithms to be efficient, the complex should have as few simplices as possible [14]. This is often achieved by a coarse-graining procedure that reduces the point cloud to a subset of points called landmarks, not without some loss of information which may, or may not, be significant. But in the context of dynamical reconstructions from chaotic-like time series, point clouds in state space are structured in a particular way, which is well described by a branched manifold. This property can be harnessed to construct a complex that has one cell per patch with two immediate benefits: the number of cells is drastically reduced, and the cell dimension respects -by construction- the local dimension of the branched manifold. The price to pay is that such a cell has a non simplicial nature, but nothing prevents the computation of the homology groups of a non simplicial complex. This approach, called Branched Manifold analysis through Homologies (BraMAH), was first reported in [23], and described in detail in [24]. BraMAH calculates homologies keeping track of the information that is required to identify the mutual organization of the branches in a branched manifold, as well as the localization of twists or torsions, thanks to the supplementary computation of orientability chains. BraMAH is of course less efficient and general than optimized packages based on bringing a complex to its canonical form by upper-triangular matrices [25], but its specificity is interesting because it provides information that is otherwise absent from the output. This work will focus on BraMAH, but one methodology does not rule out the other: simplicial and non simplicial approaches can be used in tandem if 
necessary, since their strengths are complementary.

Parallel to the proliferation of topological techniques in nonlinear dynamics, a variety of mathematical methods are being proposed to characterize the kinematics of a fluid flow from sparse data [26, 27]. These works include topology, through braid theory [28], but also other branches of mathematics such as graph theory [29]. These methodologies are expected to be particularly useful in geophysical problems [30], where Lagrangian coherent structure detection is an issue of great interest [31]. Using BraMAH to analyze Lagrangian time series will have points in common with the braid method through the use of topology [28], and with the graph theory method [29] in the evaluation of properties of individual particle trajectories. In all these approaches, the aim is to achieve a characterization of the fluid relying on the advection of a relatively sparse set of Lagrangian particles and without a knowledge of the full velocity field, which is often unavailable. A topological analysis of dynamical reconstructions from Lagrangian time series will sort trajectories in terms of the dynamics deployed in their finitetime behavior. Dynamical diversity has an important role in the formation of Lagrangian coherent sets $[32,33,34]$ which are most often defined in terms of the exchange of fluid across a transport barrier in the presence of small noise or diffusion. But they are also defined in terms of a maximal dynamical disconnection in phase space over a specified time duration of finite length [32]. Non communicating regions in a fluid can certainly be expected to have differentiated dynamics that BraMAH may be able to detect considering a set of individual particle trajectories, a fact that makes BraMAH relevant and complementary to other fluid flow characterization strategies [35, 36].

This work explores the topology of dynamical reconstructions from univariate time series associated to individual particle trajectories in finite-time windows using BraMAH. Section 2 provides basic definitions that will be useful in the rest of the article. Section 3 describes the BraMAH methodology, and section 4 presents its application in a Lagrangian context through two paradigmatic flow kinematic models: the driven Double Gyre and the Bickley Jet. Conclusions are drawn in the last section.

\section{DEFINITIONS}

\subsection{Cell and cell complex}

A few definitions may be useful before we proceed. In algebraic topology, an $n$-cell $\left(n \in \mathbb{N}_{0}\right)$ in a cell complex $\mathbb{K}$ is a set that can be mapped through a continuous invertible map into the interior of an $n$-disk, and has its boundaries 
divided into finite numbers of lower-dimensional cells, called faces. A point is a 0-cell, a line segment joining two points is a 1-cell, and so forth.

A cell complex is a finite set of cells such that the faces of the cells are elements of the complex, and such that the interiors of two cells in a complex do not intersect. The dimension $h$ of a cell complex $\mathbb{K}$ is the dimension of its highestdimensional cell.

If all cells in a complex have the minimal number of faces corresponding to the cell dimension, the cell complex is said to be simplicial, and its cells are called simplices. Non simplicial complexes or cell complexes tout court are complexes for which cells need not be simplices.

A cell complex is said to be directed or oriented if each cell is given a direction.

\subsection{Homology groups and orientability chains}

Algebraic operations enable a characterization of the topological properties of $\mathbb{K}$ in terms of homology groups. The output is layered in $k$ sets labeled $H_{k}$ with $k=0, \cdots, h$. Each of these sets are formed by elements that can be expressed as chains of $k$-cells. In a directed complex, a $k$-chain is a linear combination of $k$-cells where the coefficients are integers. These chains contain information regarding the connectivity of the cells at each $k$ level, identifying $k$-order holes in the complex.

A $k$-order hole in a cell complex is a $k$-cycle (loop or closed chain) that is not the border of a higher-dimensional cell. The $k$ th Betti number $B_{k}$ is the rank of $H_{k}$ : $B_{k}=\operatorname{dim}\left(H_{k}\right)$. The set $B_{0}$ provides the number of connected components of the complex. If the complex is made of one piece, $B_{0}$ will be 1 . If there are $p$ pieces, $B_{0}=p$ and $H_{0}$ will contain the homologically independent 0 -cells identifying each of these pieces. Similarly, $B_{1}$ indicates the number of closed paths encompassing holes, and $H_{1}$ identifies in terms of the 1-chains of the complex, the homologically independent sequence of segments encircling each of these holes. The number of enclosed cavities is provided by $B_{2}$, and the identification of the enclosed cavities in terms of homologically independent 2-cells in the complex, by the generators of $\mathrm{H}_{2}$.

If a cell complex is uniformly directed, i.e. if the orientation of every cell is the same as its neighboring cells, one can extract information regarding torsions and their location in the complex. This is achieved through the computation of orientability chains, i.e. of the chains summing all the borders of the complex that present integer multiples of cells. 


\subsection{Manifolds and branched manifolds}

A manifold is a topological space in which every point has a neighborhood that is homeomorphic to either a full $n$-ball or a half $n$-ball, in order to permit manifolds with boundaries [16].

A branched manifold is a structure, relative to a flow in state space, which allows a point cloud to be collapsed onto a lower-dimensional subset of state space $[37,38]$. This mathematical object is a manifold everywhere but at the tear points of the branches, which are precisely associated to the stretching and squeezing mechanisms that are present in the flow [39].

Let us introduce the term finite-time branched manifold to refer to branched manifolds that are strictly relative to the finite time series used in a dynamical reconstruction.

\subsection{Dynamical reconstructions and observability}

Observability is a concept that is introduced to check whether the measured variables provide a reliable characterization of the system's dynamics or not [40, 41, 42].

A measured variable is said to have full observability of the original space when there is no loss of information in the measurements. An observability analysis may therefore ensure that all the properties of the dynamics are actually "seen" by the variable.

For this reason, even if a measured time series is sufficiently long and well sampled, dynamical reconstructions cannot be guaranteed to be full without a prior knowledge of the system and its observability properties. In multivariate problems, some variables may carry more information about the whole state space dynamics than others.

A finite-time branched manifold analysis should therefore not be interpreted as global or full, but as a limited description of the underlying dynamics. Such a partial result can however be fruitful and meaningful, as will be shown in this work, when a collection of Lagrangian time series are examined. A study of observability of bidimensional fluid flows in state space can be found in [43].

\section{METHOD}

BraMAH is a method conceived to extract the topological structure of a reconstructed flow in state space from data, and therefore to characterize the main features of the finite-time dynamics contained in the dataset that is taken as input. The starting point of the analysis is a time series, or a collection of time 
series, with certain characteristics (sampling rate, length, noise level, etc). This section will describe the data requirements, the steps that must be accomplished to compute the topological properties associated to a time series, and the particular features that are encountered when dealing with Lagrangian data.

\subsection{Data requirements}

In [39], data requirements for the input of a topological analysis from time series are expressed in terms of quasi-cycles, where a quasi-cycle can be estimated as the lowest time-delay peak in a close-returns histogram, or as the inverse of the highest frequency peak in the power spectrum. In the examples considered therein [39], one hundred quasi-cycles is found to be more than enough for a topological analysis: the first dozen quasi-cycles already outline the structure of the branched manifold. Fewer than fifty samples per quasi-cycle often requires the data to be interpolated or smoothed. The generality of these thresholds can certainly not be assessed, but can be used as reference values.

In the case studies that will be considered below, the indications provided in [39] apply and are useful to draw interesting conclusions. Let us mention that in the present work, the datasets are generated with kinematic model equations, so that one can freely fix many parameters, including the length and sampling rate of the simulated time series. This will of course not be the case when dealing with datasets issued from observations or experiments. The important message here is that datasets of an "acceptable" quality and length are required for a BraMAH analysis to be feasible. A suitable preprocessing of the data may also be critical to attain optimal topological results with the available measurements.

\subsection{Computational steps}

Two separate steps must be accomplished:

- Embedding the time series in a domain that provides a suitable state-space reconstruction of the underlying dynamics,

- Computing the topological properties associated to the point cloud formed by the embedded time series.

Let us start by giving details on the first step. Time-delay embeddings are the most well-established strategy for obtaining the dynamics of a system from scalar time series [19]. Differential or integral embeddings may be preferred when the scope is to extract modeling equations from data, as done in global modeling techniques $[44,45]$, but the user must beware that an order of magnitude in the signal-to-noise ratio is often lost each time a derivative (or integral) is taken. Loss 
of two orders of magnitude degrades the embedding to the extent that the topological organization may be difficult to compute [39].

The delay coordinate reconstruction parameters can be chosen following the customary heuristics, e.g. false nearest neighbors (FNN) to determine the embedding dimension $m$, and average mutual information (AMI) to determine the time delay $\tau[46,47,48,49,50]$. In case of noisy data, the user must remember that the embedding dimension is often over-estimated by these methods [14].

The variable chosen to construct the embedding will also be relevant. In Lagrangian studies, and depending on the effective physical space dimension of the fluid system, there will be more or less spatial coordinates involved. Variables with a bad observability may involve working in higher state space dimensions, especially when the aim is to achieve a full characterization of the system dynamics. This is the case for modeling purposes, to extract models from data, or to validate a model $[44,45]$, but for classification purposes, one can remain at lower precision levels. In practice, the level of detail will be case-dependent, and must be evaluated in terms of the available variables and the particular needs.

To sum up, the first step of the analysis implies choosing (i) a scalar variable $v$, (ii) a time window $T_{w}$, (iii) an embedding dimension $m$, and (iv) a time delay $\tau$. For topological comparison and classification purposes, these four choices should be kept fixed for the time series collection under inspection.

Let us now turn to the second step of the analysis. Topological feature extraction from delay-embedded time series is revisited in [14]. In that work, the extraction of the large-scale topological features of a state-space point cloud are found to be affordable with low-dimensional embeddings, i.e. of a dimension that is lower than the one required for a full reconstruction of the dynamics. This point cloud can be placed on a single geometric object that operates as a point-cloud organizer or template of the embedded time series. Mathematically, this object is a finite-time branched manifold, the term introduced in 2.3 to stress that it is obtained from a finite time dataset.

The approach adopted in this work is built upon the algorithm described in [24]. BraMAH builds non simplicial complexes, i.e. complexes for which cells need not be simplices. Given a point cloud $\left\{\boldsymbol{x}_{\boldsymbol{i}} / \boldsymbol{x}_{\boldsymbol{i}} \in \mathbb{R}^{m} \wedge 1 \leq i \leq N\right\}$, the non simplicial complex constructed with the BraMAH procedure requires a prior decomposition of the point cloud into overlapping point sets called patches, defined as homeomorphic to the interior of a $d$-disk, $d$ being the local dimension of the branched manifold $(d \leq m)$. Intersections between patches allow keeping track of the gluing prescriptions that are necessary to assemble them. Each patch is associated to a point $\boldsymbol{x}_{\mathbf{0}}$ that is used as center or axis of the disk that encloses 
its neighbors. The size $N_{c}$ of the patch $\left\{\boldsymbol{x}_{\boldsymbol{i}}, i=1, \cdots, N_{c}\right\}$ around a center $\boldsymbol{x}_{\mathbf{0}}$ is computed searching for the largest number of points around $\boldsymbol{x}_{\mathbf{0}}$ that constitute good approximations to a Euclidean set. The search is done within limits $N_{\text {min }}$ and $N_{\text {max }}$ that are selected so that the point density is approximately uniform. A series of candidate sets $\left\{\boldsymbol{x}_{\boldsymbol{i}}, i=1, \cdots, n_{c}\right\}$ are considered, with its elements sorted in order of increasing distance from $\boldsymbol{x}_{0}$, and with $n_{c}$ sweeping the values between the limits $\left(N_{\min }, N_{\max }\right)$. The criterion used to choose the best candidate, i.e. to find $N_{c}$, is the same used in [16,51], and can be synthesized as follows.

Given a center $\boldsymbol{x}_{\mathbf{0}}=\left(x_{0,1}, x_{0,2}, \cdots, x_{0, m}\right)$, all the points $\boldsymbol{x}_{\boldsymbol{i}}=\left(x_{i, 1}, x_{i, 2}, \cdots, x_{i, m}\right)$ that lie within the ball centered at $x_{0}$ and radius $r$, are represented by the neighborhood matrix $X \in \mathbb{R}^{n_{c} \times m}$

$$
X_{i, j}=\frac{1}{\sqrt{n_{c}}}\left(x_{i, j}-x_{0, j}\right)
$$

The singular vectors of $X$ give a local coordinate system centered at $\boldsymbol{x}_{\mathbf{0}}$, and the singular values describe the distribution of the points within the ball centered at $\boldsymbol{x}_{\mathbf{0}}$. For small enough $r$, the effects of curvature of the $d$-dimensional patch of the manifold become unimportant and the patch is well approximated by its tangent space at $\boldsymbol{x}_{0}$. The local singular spectrum of $X$ for a $d$-manifold in $\mathbb{R}^{m}$ will have $d$ singular values that will scale linearly with $n_{c}$ as $r$ is increased, until saturation or the effects of curvature in the manifold become noticeable. The remaining $(m-d)$ singular values will measure the deviation from the tangent space of the manifold and will scale, due to the curvature, as $r^{2}$ or as higher powers of $r$. This property allows us to distinguish the $d$ relevant singular values from the $(m-d)$ non relevant ones: $n_{c}=N_{c}$ when the $d$ largest singular values of $X$ present the best linear regression coefficient. After this process, the $d$-dimensional patch is the set $\left\{\boldsymbol{x}_{\boldsymbol{i}}, i=1 \ldots N_{c}\right\}$. Once the whole point cloud is decomposed into patches, convex hulls are used to construct a uniformly directed complex in which each cell corresponds to a patch. The result is a non simplicial complex $\mathbb{K}$ with dimension $h=d$, and with a number of cells that is considerably lower than the number of points in the cloud.

The next step in BraMAH is to extract homology groups and relevant torsion information from the constructed complex. The symbolic code in Wolfram Mathematica written in $[23,24]$ was updated and used to extract the homology groups and orientability chains from the non simplicial uniformly directed cell complex that is constructed in the previous step. The code performs the following operations: (i) it reads the list of vertices in order to construct a boundary map matrix; (ii) it extracts the linearly independent rows of the boundary map matrices; (iii) 
it computes the null spaces of the transpose of the boundary map matrices; (iv) it expresses the $k$-borders in terms of the the $k$-cycles in order to find which $k$-cycles are homologous to others; (v) it appends in $H_{k}$ the $k$-cycles that are homologically independent; and (vi) it identifies the cells preceded by integer multiples in the chains summing all the borders. The output consists of the homology groups $H_{k}$ spelled in terms of their generators, and the orientability chains, if there are any. Because the cells of the complex are denoted using a list of labeled vertices corresponding to numbered points in the original cloud, the generators can be used to identify the branches in the cloud, and the orientability chains to locate the existence of twists.

\subsection{Lagrangian time series}

Let us consider the application of this methodology to Lagrangian time series presenting some kind of recurrent bounded behavior in reconstructed state space, and satisfying the data requirements described in 3.1.

When a collection of time series under analysis is Lagrangian, or in other words, associated to the motion of a fluid particle, the results of the BraMAH analysis described in 3.2 can be represented, not only in the reconstructed state space, as is customary, but also in the spatial domain where fluid particles flow.

All time series sharing the same topological structure, namely the same organization of branches and torsions, can be assigned a common label, and that label can be located in physical or 'geographical' space in correspondence with the particle that is associated to the inspected time series. In analytical cases, as the ones we will be considering in this work, one can introduce the usage of what we shall term, for short, topological grids. In this representation one finds at each position of the grid, the label corresponding to the topological class of a particle that is passing through that position at an instant $t_{s}$ for a time series defined for instance in $\left[t_{s}, t_{s}+T_{w}\right]$ (or $\left[t_{s}-T_{w}, t_{s}\right]$ ). Notice that a topological grid is always defined in relation with a time $t_{s}$ and a window $T_{w}$. If the grid is sufficiently fine, the temporal evolution of the labels will show how the distribution of topological classes changes in space as $t_{s}$ is varied.

\section{RESULTS}

In this section, we show the results of the analysis of Lagrangian time series generated with two analytical nonlinear kinematic flow models, typically used as testbench in Lagrangian studies: the driven Double Gyre and the Bickley Jet [29]. The driven Double Gyre is a bidimensional cellular flow inspired in oceanic flow 
patterns proposed by [52], and the Bickley Jet is an example of a two-dimensional open flow, frequently used to model zonal jets in the Earth's atmosphere [53, 54].

Both models are defined in terms of a stream function $\psi(x, t)=\psi\left(x_{1}, x_{2}, t\right)$, so that the motion of the fluid particles located at $x(0)$ evolve according to:

$$
\dot{x}=V(x, t)
$$

with $V=\left(\frac{\partial \psi}{\partial x_{2}},-\frac{\partial \psi}{\partial x_{1}}\right)$. These ordinary differential equations can be numerically integrated to generate individual particle trajectories that will serve as input for BraMAH.

The interest here is to illustrate how the BraMAH approach performs on these well-known cases. In both cases, the analyses will be conducted on a collection of time series for sparsely distributed particles at a certain time step. The output will allow for a classification of the different time series according to the homologies and orientability properties of the cell complex. Topological grids will be constructed with these results, in order to visualize the spatial organization of the topological results.

Topological grids will be presented in juxtaposition with streakline snapshots. Dye continuously injected into a fluid at a fixed point extends along a streakline. In practical situations, this may correspond to different scale problems ranging from oil spills in the ocean or to the injection of small amounts of drugs from small tubes in cells of microfluidic devices. Streaklines are used in the laboratory to visualize flows, to develop flow control strategies [55] or to analyze mixing [56]. Computing a streakline for a kinematic model amounts to conducting a virtual experiment in which dye is continuously released from a fixed location, called seeding or injection point. Streakline snapshots show the dyed particles at a certain instant. We will use streaklines to compare the results of the BraMAH analysis on individual particle trajectories with the transport properties of the flow.

\subsection{Driven Double Gyre}

The driven Double Gyre system [52] is defined by the stream function $\psi$, the domain under consideration is $\Omega=[0,2] \times[0,1]$ and usual parameter values are $A=0.1, \eta=0.1$ and $\omega=\pi / 5$ :

$$
\psi\left(x_{1}, x_{2}, t\right)=A \sin \left(\pi f\left(x_{1}, t\right)\right) \sin \left(\pi x_{2}\right)
$$

with:

$$
f\left(x_{1}, t\right)=a(t) x_{1}^{2}+b(t) x_{1}
$$




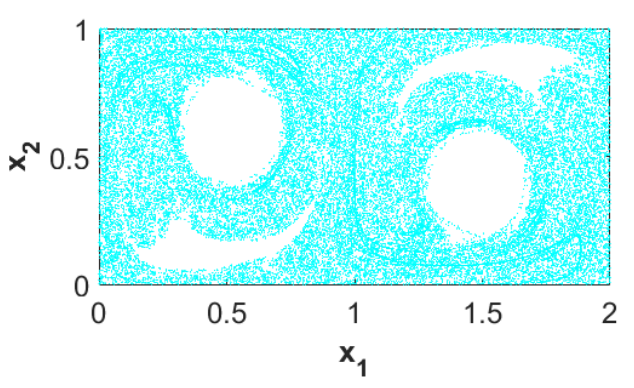

(a)

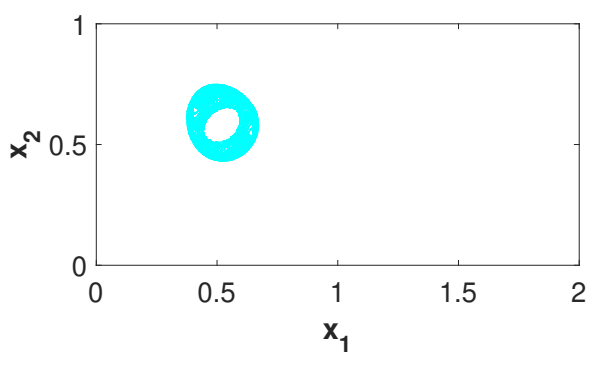

(b)

Figure 1: Streakline images in the driven Double Gyre flow $(A=0.1, \eta=0.1$ and $\omega=\pi / 5)$ at time $35 T_{p}$, with $T_{p}=2 \pi / \omega$. Injection points are: (a) $\mathbf{p}=(1,0.5)$ and (b) $\mathbf{p}=(0.5,0.5)$.

$$
\begin{gathered}
a(t)=\eta \sin (\omega t), \\
b(t)=1-2 a(t) .
\end{gathered}
$$

Let us first characterize the transport properties of this flow using streaklines. Figure 1 shows streakline images with different injection points: (a) at $\mathbf{p}=(1,0.5)$ and (b) at $\mathbf{p}=(0.5,0.5)$. The elapsed time in both cases is long enough for the tracer to invade all the reachable spaces of the domain ( $35 T_{p}$ with $\left.T_{p}=2 \pi / \omega\right)$. In (a), one can observe four closed regions or islands in which the tracer does not penetrate. These islands adopt either a circular or an arrow-like shape; they are not static and rotate in each half domain suffering deformation in their shape during their displacement, keeping the same size due to incompressibility [57]. When the injection is performed in the left circular blank island of (a), as shown in (b), the tracer never invades the central part of the circle. These images illustrate the well-known presence of regions in the driven Double Gyre flow, which experience no mixing with the surrounding fluid.

Let us now perform a BraMAH analysis for particle trajectories generated with this system. For the sake of demonstration, the Lagrangian time series are generated with characteristics that largely satisfy the technical requirements described in section 3.1: the time window has a length $T_{w}=500$ and the sampling rate is $s_{r}=T_{p} / 100=0.1$. Concerning the variable choice, we will show the method at work on the horizontal position coordinate, labeled $x_{1}$, which is more interesting than the vertical position coordinate for symmetry reasons. Figure 2 shows four individual particle trajectories initiated at different positions with their respective time series. 

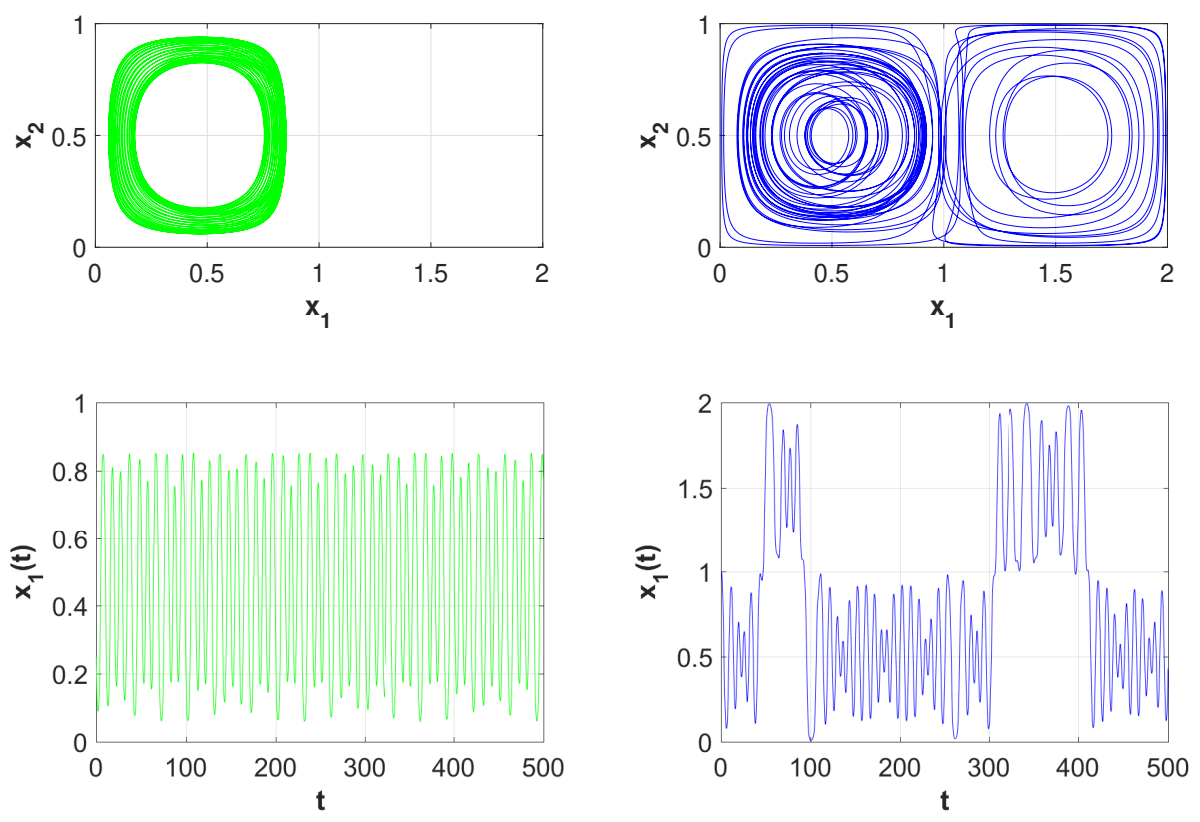

(1)

(2)
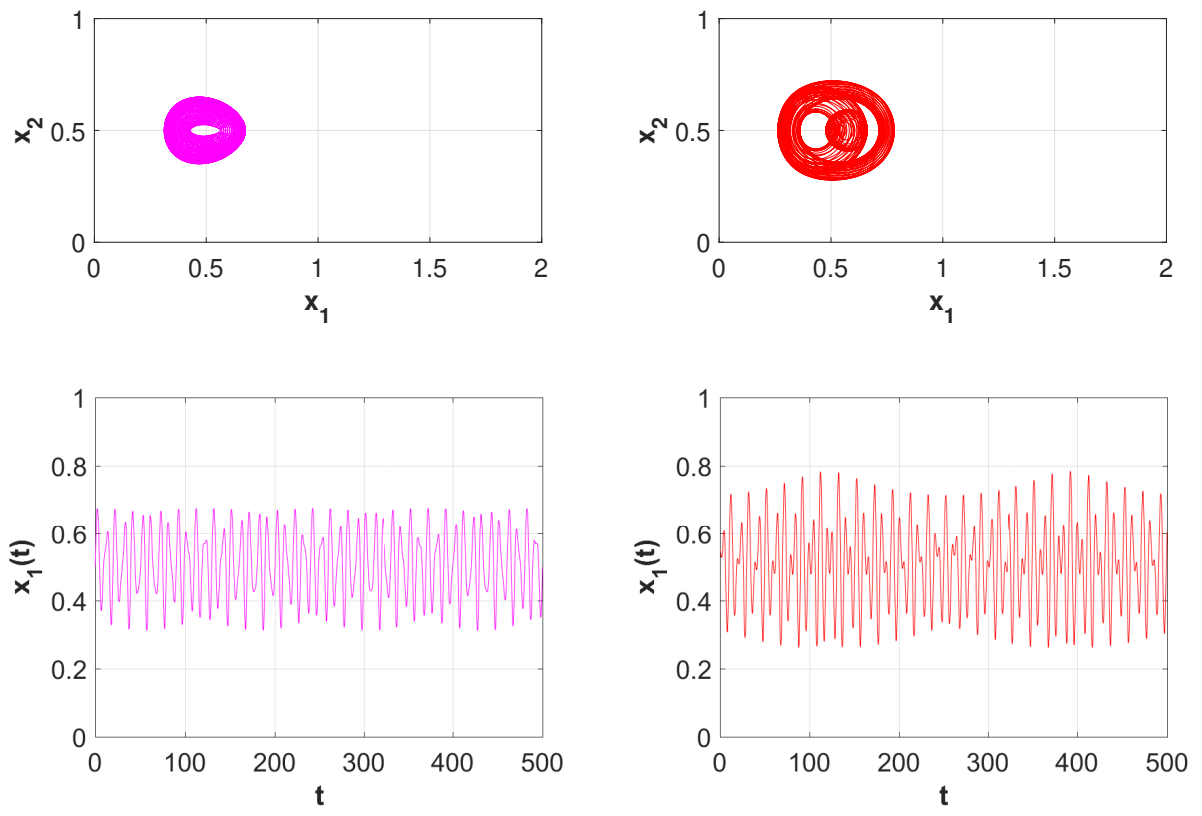

(3)

(4)

Figure 2: Individual particle trajectories of the driven Double Gyre and associated $x_{1}$ time series used as input in the BraMAH analysis for different initial positions: (1) $\mathbf{p}_{01}=(0.25,0.125)$, (2) $\mathbf{p}_{02}=(1,0.5),(3) \mathbf{p}_{03}=(0.5,0.625),(4) \mathbf{p}_{04}=(0.55,0.5)$. 


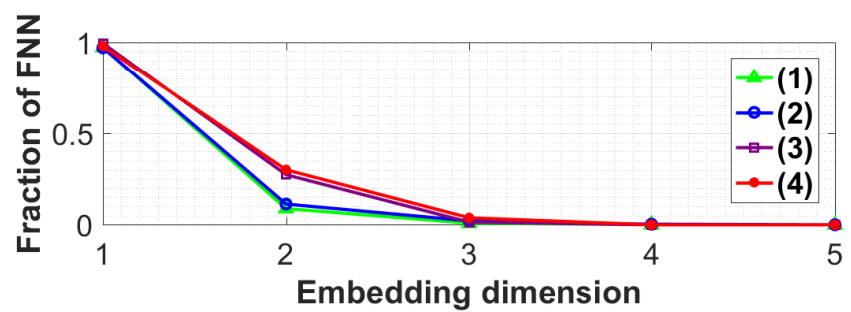

Figure 3: Fraction of false nearest neighbors (FNN) as a function of the embedding dimension for the driven Double Gyre time series (1), (2), (3), (4) shown in Figure 2.

The first step is to select the parameters for the time-delay reconstruction. An FNN test on these time series shows that the reconstruction dimension stabilizes at an embedding dimension of $m=4$, as shown in Figure 3. The delay $\tau$ is chosen among the minima indicated by AMI tests [47] and is set to $\tau=0.2 T_{p}$.

The results of the BraMAH analysis of the four time series of Figure 2 are shown in Figure 4. With these settings, the four-dimensional point clouds that result, define finite-time branched manifolds that are locally bidimensional $(d=$ 2 ), and that can be therefore approximated by complexes $\mathbb{K}$ with dimension $h=2$. Three-dimensional projections of the four-dimensional point clouds are shown together with the non simplicial complexes generated for each point cloud by the algorithm detailed in Section 3.2. The homology groups of each of the four cell complexes are:

(1) Standard strip: $H_{0}\left(\mathbb{K}_{1}\right) \sim \mathbb{Z}, H_{1}\left(\mathbb{K}_{1}\right) \sim \mathbb{Z}, H_{2}\left(\mathbb{K}_{1}\right) \sim \emptyset$,

(2) Five-handle structure with a torsion: $H_{0}\left(\mathbb{K}_{2}\right) \sim \mathbb{Z}, H_{1}\left(\mathbb{K}_{2}\right) \sim \mathbb{Z}^{5}$ with one orientability chain, $H_{2}\left(\mathbb{K}_{2}\right) \sim \emptyset$,

(3) Torus: $H_{0}\left(\mathbb{K}_{3}\right) \sim \mathbb{Z}, H_{1}\left(\mathbb{K}_{3}\right) \sim \mathbb{Z}^{2}, H_{2}\left(\mathbb{K}_{3}\right) \sim \mathbb{Z}$,

(4) Moebius strip: $H_{0}\left(\mathbb{K}_{4}\right) \sim \mathbb{Z}, H_{1}\left(\mathbb{K}_{4}\right) \sim \mathbb{Z}$ with one orientability chain, $H_{2}\left(\mathbb{K}_{4}\right) \sim \emptyset$

Complexes $\mathbb{K}_{1}$ and $\mathbb{K}_{4}$ have a single 1-hole and complex $\mathbb{K}_{3}$ encloses a single 2-hole, i.e. a cavity. The $H_{1}$ generators for each complex $\mathbb{K}_{i}$ with $i=1,2,3,4$ are 
illustrated in color on the cell complexes. Torsions are detected only in complexes $\mathbb{K}_{2}$ and $\mathbb{K}_{4}$ of Figure 4 . As the topology of $\mathbb{K}_{1}$ and $\mathbb{K}_{4}$ is single-hole $\left(H_{1} \sim \mathbb{Z}\right)$, the presence of a torsion in $\mathbb{K}_{4}$ provides a distinction from $\mathbb{K}_{1}$ which would otherwise be undetectable. This shows the importance of computing orientability chains along with homology groups.

Looking at Figures 2 and 4 simultaneously, one can remark that the time series associated to the strips and the torus correspond to particles that remain in only one hemisphere of the domain, while the five-handle structure corresponds to a background flow trajectory, that visits both hemispheres.

Let us now enlarge the dataset under analysis with particles located at other initial positions in the domain. We will consider collections of $N_{p}=105$ particles with sparsely distributed initial positions. Each of the 105 time series is found to fall into one of the four topological categories already presented in Figure 4. The Moebius-strip class of trajectories presents a particular feature: the point clouds are such that one can distinguish lanes along the branches. The banded structure of a branched manifold can be studied in terms of subtemplates [58]: a BraMAH analysis can be conducted to describe these subtemplates adjusting $\left(N_{\min }, N_{\max }\right)$ which set the approximate size of the cells, but the level of detail required to discriminate subtemplates is out of the scope of the current analysis. All these particle trajectories are therefore classified within (4), independently of the existence of subclasses at a finer scale.

In order to construct the topological grids, let us introduce the following symbols: triangles $(\triangle)$ for $\mathbb{K}_{1}$, circles $(\bigcirc)$ for $\mathbb{K}_{2}$, squares $(\square)$ for $\mathbb{K}_{3}$, and asterisks (*) for $\mathbb{K}_{4}$. The $N_{p}$ initial particle positions will be plotted using the symbol that corresponds to the corresponding result of the BraMAH analysis. As anticipated, the topological grid is shown in juxtaposition with streakline snapshots used for reference. Figure 5 shows four snapshots of the topological grids of the flow for the streakline having $\mathbf{p}=(1,0.5)$ as injection point. The sequence provides a clear idea of the evolution of the flow regions, and of the topological classes observed for particles placed at different positions. A complementary plot is provided in Figure 6 to show the detail inside the circular region for the streakline with injection point at $\mathbf{p}=(0.5,0.5)$.

The juxtaposition of streaklines and topological grids shows the close connection found between the branched manifold analysis of individual particle trajectories and the transport properties of the kinematic flow. There is a significant similarity between the topological grids and the patterns that are found with the braid theory and the transfer operator in [26], and with the coherent structure coloring method applied to the quadruple gyre in [59], even if the time windows of 

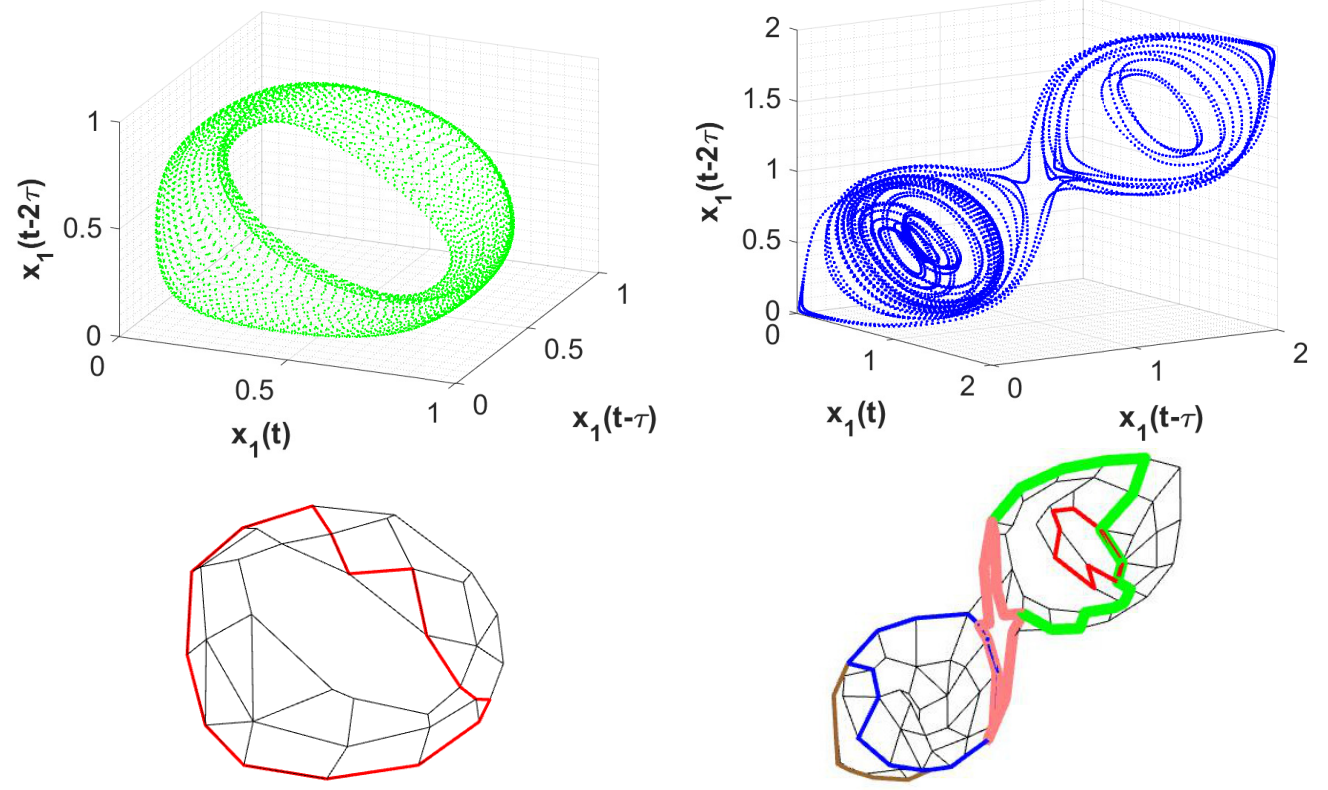

(1) $\mathbb{K}_{1}$ with $H_{1}\left(\mathbb{K}_{1}\right) \sim \mathbb{Z}$

(2) $\mathbb{K}_{2}$ with $H_{1}\left(\mathbb{K}_{2}\right) \sim \mathbb{Z}^{5}$
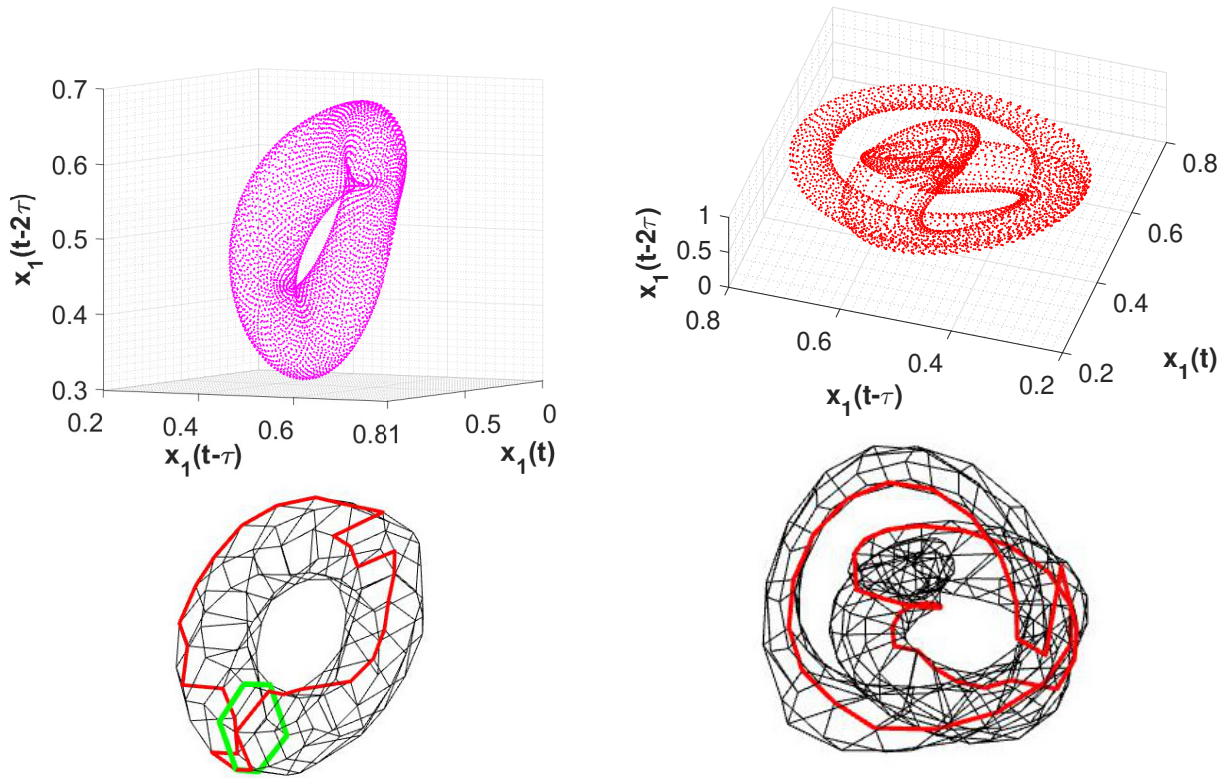

(3) $\mathbb{K}_{3}$ with $H_{1}\left(\mathbb{K}_{3}\right) \sim \mathbb{Z}^{2}$

(4) $\mathbb{K}_{4}$ with $H_{1}\left(\mathbb{K}_{4}\right) \sim \mathbb{Z}$

Figure 4: BraMAH analysis for the driven Double Gyre times series in Figure 2. Threedimensional projections of the embeddings with $\tau=0.2 T_{p}$ and $m=4$ are shown. The associated non simplicial complexes with dimension $h=2$ are presented, with the $H_{1}$ generators highlighted in color. 


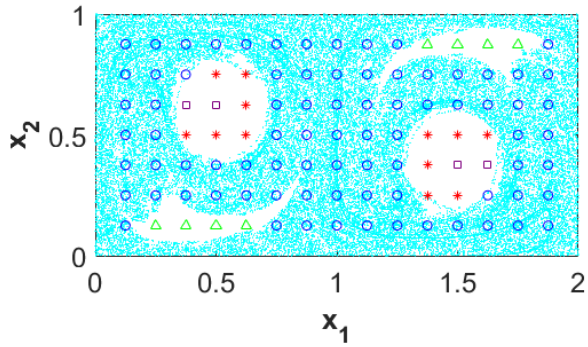

$t_{s}=39 T_{p}$

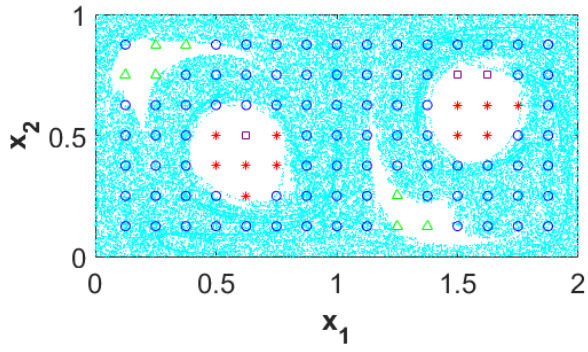

$t_{s}=39.4 T_{p}$

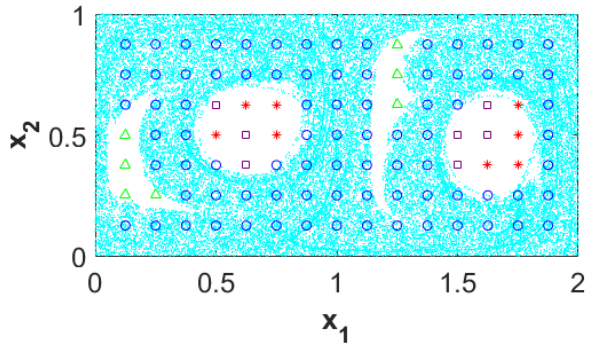

$t_{s}=39.2 T_{p}$

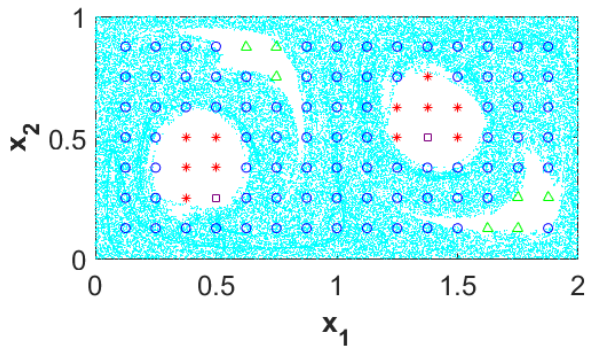

$t_{s}=39.6 T_{p}$

Figure 5: Snapshots of the driven Double Gyre flow showing the streakline injected in $\mathbf{p}=(1,0.5)$ together with the BraMAH topological grid, where $t_{s}$ is the snapshot time and topological classes are: (a) $\mathbb{K}_{1}(\triangle)$, (b) $\mathbb{K}_{2}(\bigcirc)$, (c) $\mathbb{K}_{3}(\square)$ and (d) $\mathbb{K}_{4}(*)$.

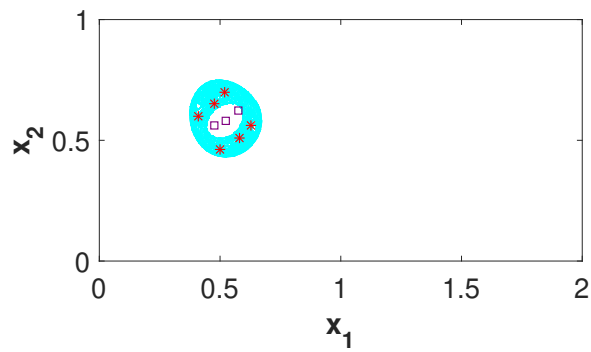

Figure 6: Streakline in the driven Double Gyre flow at time $t_{s}=35 T_{p}$ seeded at $\mathbf{p}=(0.5,0.5)$ juxtaposed with the results of the BraMAH analysis: $\mathbb{K}_{3}(\square), \mathbb{K}_{4}(*)$. 
the studies differ.

Particles in the background flow are associated to the topology of complex $\mathbb{K}_{2}$. Particles in the arrow-like islands, that orbit around the circular ones, have a dynamics corresponding to the topology of complex $\mathbb{K}_{1}$. Inside each of the two circular islands that remain out of reach for the tracer injected in the center of the domain, two different topological classes are found. The most eccentric particles correspond to the topology of complex $\mathbb{K}_{4}$, and the most central ones to the topology of complex $\mathbb{K}_{3}$, as visualized when the injection point is moved to the interior of a circular region.

The connection suggests that particles within regions that are separated by a transport barrier from the surrounding fluid are associated to the same finite-time branched manifold.

\subsection{Bickley jet}

Let us now turn to the kinematic model of an open flow. The Bickley Jet is a model gathering the essential characteristics of the stratospheric polar vortex in which a zonal jet acts as a transport barrier. The stream function $\psi\left(x_{1}, x_{2}, t\right)$ has a steady background flow $\psi_{0}\left(x_{2}\right)$ and three traveling Rossby waves are superimposed $[53,60]$,

$$
\begin{gathered}
\psi\left(x_{1}, x_{2}, t\right)=\psi_{0}\left(x_{2}\right)+\psi_{1}\left(x_{1}, x_{2}, t\right) \\
\psi_{0}\left(x_{2}\right)=-U_{0} L_{0} \tanh \left(\frac{x_{2}}{L_{0}}\right) \\
\psi_{1}\left(x_{1}, x_{2}, t\right)=U_{0} L_{0} \operatorname{sech}^{2}\left(\frac{x_{2}}{L_{0}}\right) \sum_{n=1}^{3} \varepsilon_{n} \cos \left(k_{n}\left(x_{1}-c_{n} t\right)\right) .
\end{gathered}
$$

The parameters are the characteristic velocity $U_{0}=62.66 \mathrm{~m} / \mathrm{s}$, the characteristic length scale $L_{0}=1770 \mathrm{~km}, \varepsilon_{1}=0.0075, \varepsilon_{2}=0.15, \varepsilon_{3}=0.3, l_{x}=6.3717 \times$ $10^{6} \pi, k_{n}=2 n \pi / l_{x}, c_{1}=0.1446 U_{0}, c_{2}=0.205 U_{0}$ and $c_{3}=0.461 U_{0}$.

Figure 7 shows a streakline snapshot at 40 days with injection locations $\mathbf{p}=$ $\left(x_{1}, x_{2}\right) / x_{1}=1 \times 10^{6} \wedge x_{2} \in[-4: 0.05:-2] \times 10^{6} \cup[2: 0.05: 4] \times 10^{6}$, with the length units measured in meters. The snapshot shows that this kinematic flow also presents regions with little mixing with the background fluid. The regions that are not invaded by the tracer are the sequence of counter-rotating vortices and a central alley corresponding to the sinuous jet region.

The BraMAH analysis of the Bickley Jet trajectories is performed using scalar time series of the vertical coordinate $x_{2}$, the variable that carries the information of the north and south hemispheres of the model. For demonstration purposes, 


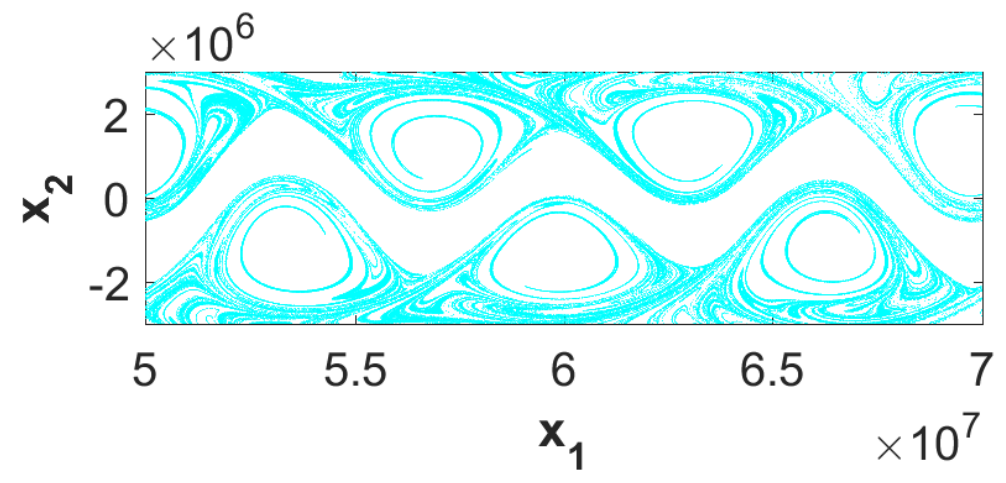

Figure 7: Streakline associated to the Bickley Jet flow at 40 days. The injection locations are points $\mathbf{p}=\left(x_{1}, x_{2}\right) / x_{1}=1 \times 10^{6} \wedge x_{2} \in[-4: 0.05:-2] \times 10^{6} \cup[2: 0.05: 4] \times 10^{6}$. Length units are meters.

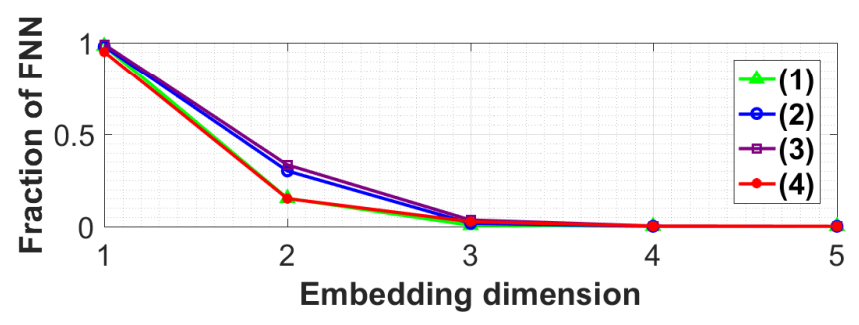

Figure 8: Fraction of false nearest neighbors (FNN) as a function of the embedding dimension for the Bickley Jet. 

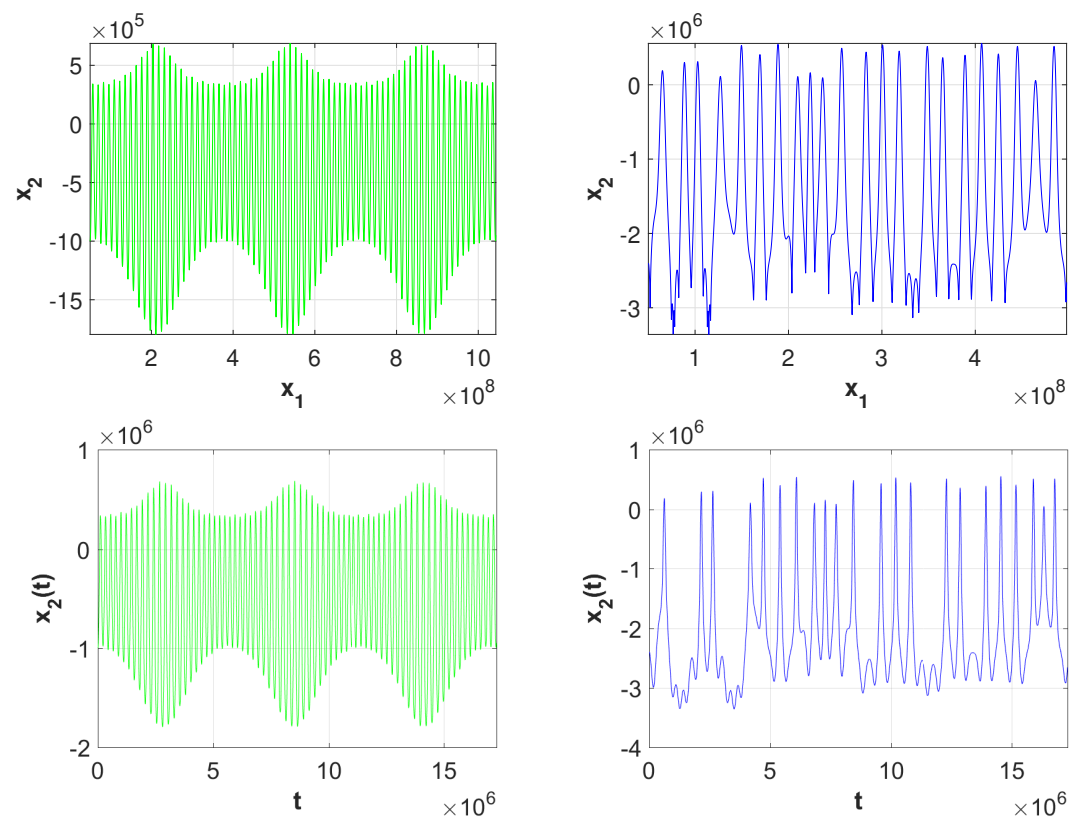

(1)
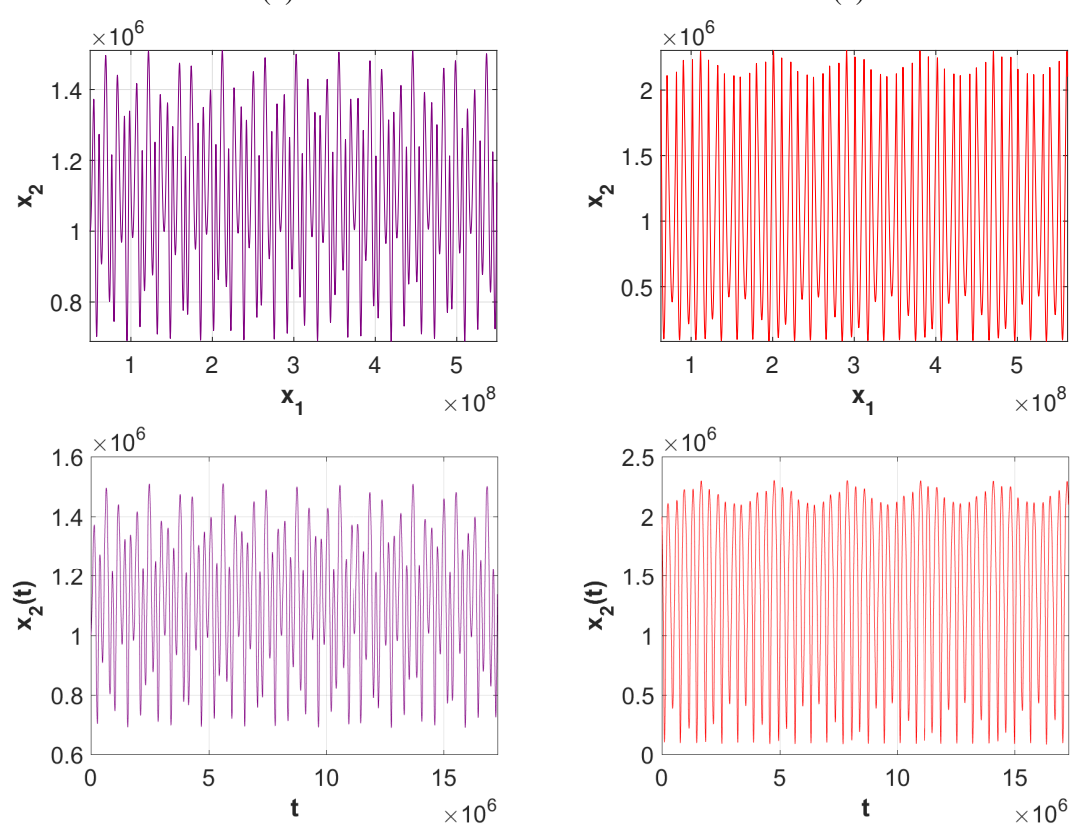

(3)

(4)

Figure 9: Individual particle trajectories of the Bickley Jet, and associated $x_{2}$ time series with $t$ in seconds and $x_{2}$ in meters, used as input in the BraMAH analysis for different initial positions: (1) $\mathbf{p}_{01}=\left(6 \times 10^{7}, 1 \times 10^{6}\right),(2) \mathbf{p}_{02}=\left(6 \times 10^{7}, 2 \times 10^{6}\right),(3) \mathbf{p}_{03}=\left(6 \times 10^{7},-1 \times 10^{6}\right),(4) \mathbf{p}_{04}=$ $\left(6.3 \times 10^{7}, 2 \times 10^{6}\right)$. 


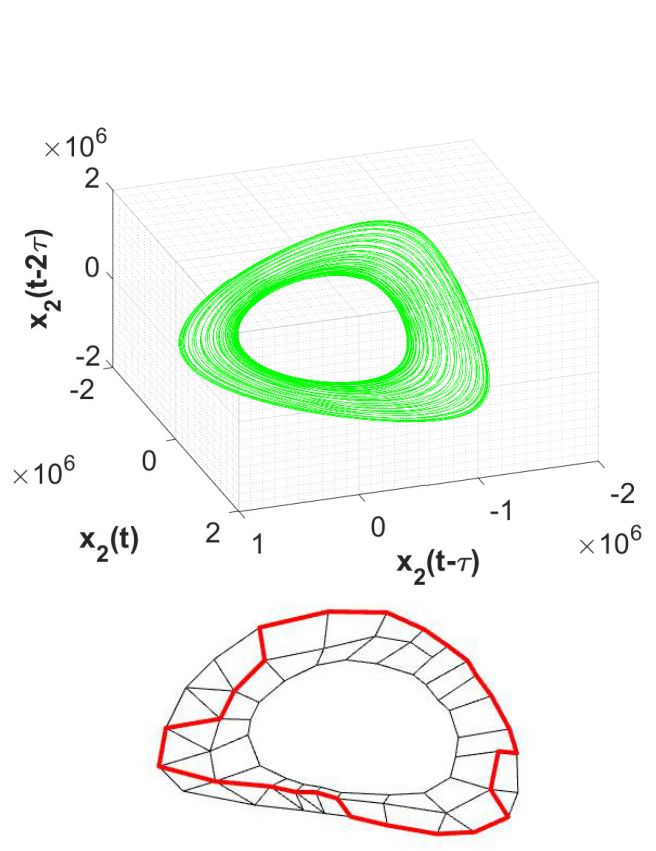

(1) $\mathbb{K}_{1}$ with $H_{1}\left(\mathbb{K}_{1}\right) \sim \mathbb{Z}$

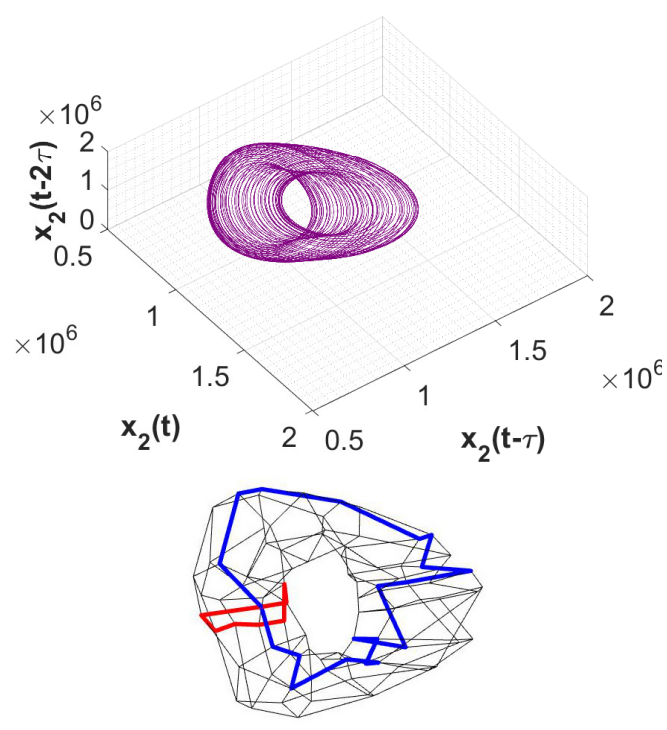

(3) $\mathbb{K}_{3}$ with $H_{1}\left(\mathbb{K}_{3}\right) \sim \mathbb{Z}^{2}$

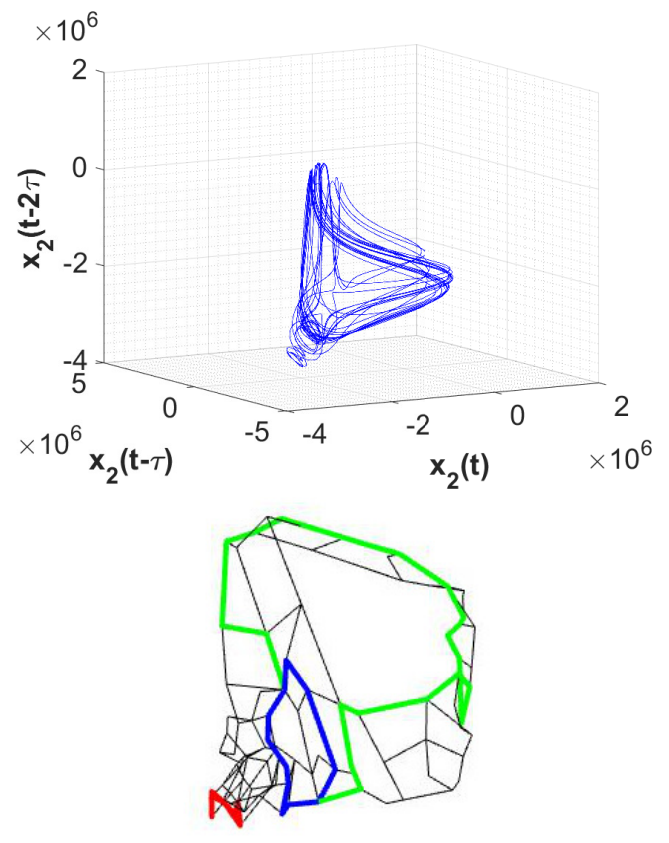

(2) $\mathbb{K}_{2}$ with $H_{1}\left(\mathbb{K}_{2}\right) \sim \mathbb{Z}^{3}$

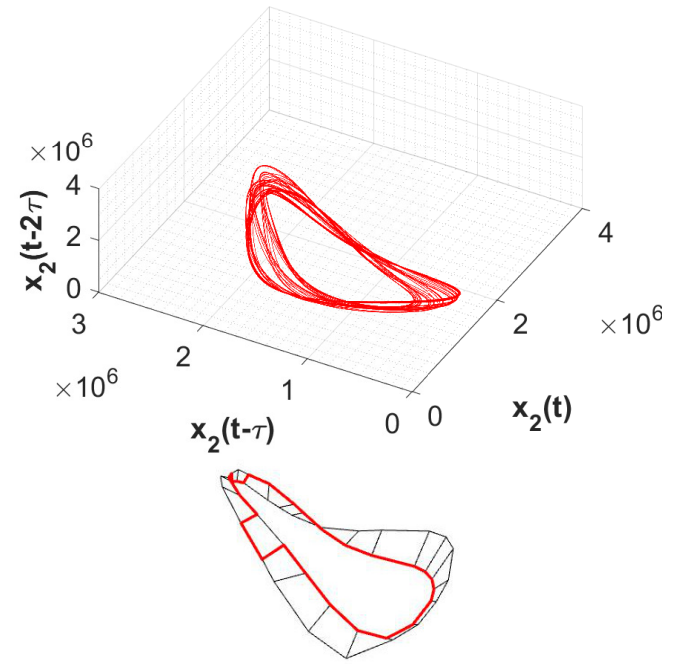

(4) $\mathbb{K}_{4}$ with $H_{1}\left(\mathbb{K}_{4}\right) \sim \mathbb{Z}$

Figure 10: BraMAH analysis of the Bickley Jet the times series in Figure 9. Three-dimensional projections of the embeddings with $\tau=6 \times 10^{4}$ seconds and $m=4$ are shown. The associated non simplicial complexes with dimension $h=2$ are presented, with the $H_{1}$ generators highlighted in color. 
the time window is set to $T_{w}=200$ days, a value that is well above the minimal time series length needed for the analysis. The FNN algorithm is implemented to estimate the embedding dimension, see Figure 8, that yields $m=4$. The timedelay is fixed at $\tau=6 \times 10^{4}$ seconds. These settings yield four-dimensional point clouds defining finite-time branched manifolds that are locally bidimensional $(d=$ 2 ), and that can be therefore approximated by complexes with dimension $h=$ 2. The methodology in 3.2 is applied to time series obtained for a set of $N_{p}=$ 145 particle trajectories initiated at different positions, sparsely distributed in the spatial domain. Particle trajectories with their respective time series are shown in Figure 9. The homology computations produce the following results:

(a) Standard strip: $H_{0}\left(\mathbb{K}_{1}\right) \sim \mathbb{Z}, H_{1}\left(\mathbb{K}_{1}\right) \sim \mathbb{Z}, H_{2}\left(\mathbb{K}_{1}\right) \sim \emptyset$,

(b) Three-handle structure with a torsion: $H_{0}\left(\mathbb{K}_{2}\right) \sim \mathbb{Z}, H_{1}\left(\mathbb{K}_{2}\right) \sim \mathbb{Z}^{3}$ with one orientability chain, $\mathrm{H}_{2}\left(\mathbb{K}_{2}\right) \sim \emptyset$,

(c) Torus: $H_{0}\left(\mathbb{K}_{3}\right) \sim \mathbb{Z}, H_{1}\left(\mathbb{K}_{3}\right) \sim \mathbb{Z}^{2}, H_{2}\left(\mathbb{K}_{3}\right) \sim \mathbb{Z}$,

(d) Moebius strip: $H_{0}\left(\mathbb{K}_{4}\right) \sim \mathbb{Z}, H_{1}\left(\mathbb{K}_{4}\right) \sim \mathbb{Z}$ with one orientability chain, $H_{2}\left(\mathbb{K}_{4}\right) \sim \emptyset$

Four topology classes are identified. Figure 10 shows the results obtained for the four time series of Figure 9. The topology grids for the Bickley Jet are shown in Figure 11. The connection between the topological classes and the transport properties of the flow is again apparent when juxtaposing the topological grid with the streakline snapshot. The fourth topological class presents subclasses, which are not identified in this work, as explained in subsection 4.1. Let us remark that the topological grid is consistent with the patterns unveiled using coherent structure coloring in [29, 59].

\subsection{Compared results}

Let us briefly consider the similarities and differences in the BraMAH results between the two kinematic flows considered above. In all cases, the embedding dimension from the FNN tests is $m=4$ and the local dimension of the finite-time branched manifolds found in the patch decomposition of the point clouds is $d=2$. As a consequence, all cell complexes have dimension $h=2$.

Considering the time series collection of the two kinematic flows, a total of five non equivalent topological classes are detected, and some of them are present in the two flow case studies. A topological correspondence exists between the 


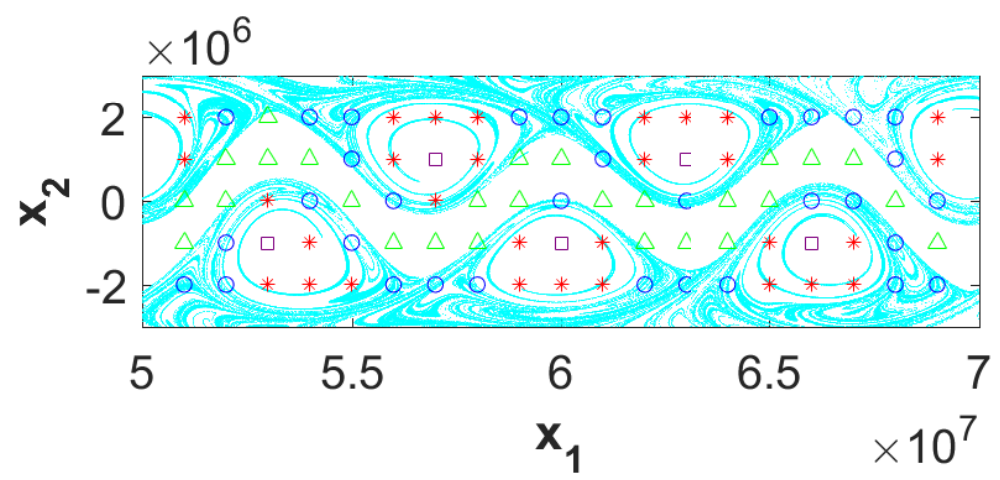

Figure 11: Streakline associated to the Bickley Jet flow at $t_{s}=40$ days with injection locations at $\mathbf{p}=\left(x_{1}, x_{2}\right) / x_{1}=1 \times 10^{6} \wedge x_{2} \in[-4: 0.05:-2] \times 10^{6} \cup[2: 0.05: 4] \times 10^{6}$, juxtaposed with the topological grid where (a) $\mathbb{K}_{1}(\triangle)$, (b) $\mathbb{K}_{2}(\bigcirc)$, (c) $\mathbb{K}_{3}(\square)$ and (d) $\mathbb{K}_{4}$ (*). Length units are measured in meters.

sinuous jet of the Bickley flow and the triangular regions of the driven Double Gyre: all these regions are topologically represented by a standard strip. The same statement holds between the vortices in the Bickley Jet and the circular driven Double Gyre regions, that are identified with Moebius strips. In the particular case of the Moebius strips, there are subclasses which are not being analyzed in detail in this work. Notice that the similarities and differences between the topological features associated to the two kinematic flow models would not be necessarily manifest through a spectral analysis.

In both systems, all particles in the background flow possess a topological structure that is richer than the one encountered in the Lagrangian islands or alleys. Inside the islands, smaller islands can be found with their own topological description. The driven Double Gyre background flow has a higher complexity -measured in terms of the number of handles- with respect to the Bickley Jet results. 


\section{Conclusions}

This work explores the topology of dynamical reconstructions from Lagrangian time series using Branched Manifold analysis through Homologies (BraMAH). The study is carried out in the context of nonlinear kinematic flow models using, for demonstration purposes, two models that are canonical in Lagrangian studies: the driven Double Gyre and the Bickley Jet.

Applying BraMAH to a collection of time series associated to individual trajectories of sparse fluid particles, distinct topological classes are identified. Topological grids are built to show how the topological classes are distributed in the flow domain. The arrangement of the topological classes in these grids are found to be related with the regions attained (or not) by a continuously injected tracer (streakline). These results suggest that particles moving in regions with poorlymixing properties have a dynamics that differs, through their state-space topology, from those in the surrounding fluid.

To conclude, this work shows that the BraMAH approach can be used to classify fluid particles in terms of the topology of the finite-time branched manifolds associated to them. No prior knowledge on the fluid flow is necessary, and the individual particle trajectories need not be closely spaced. The time series chosen for the analysis must exhibit some kind of recurrent behavior, that needs not be periodic. The sampling rate and the time-window length must be large enough for the dynamical reconstruction to be possible. For datasets in which these technical requirements are met, BraMAH constitutes a complement of other sparse flow characterization methods, with promising perspectives in Lagrangian studies.

Acknowledgements: Insightful discussions with Prof. Michael Ghil are acknowledged. G. D. C. acknowledges support of her Ph.D. thesis by CONICET. This work is supported by the MATH-GEO (18-MATH-04) project of the Regional Program MATH-AmSud.

\section{References}

[1] S. Novikov, Henri Poincaré and XXth century topology, in: In Solvay Workshops and Symposia. Symposium Henri Poincaré, edited by P. Gaspard, M. Henneaux, and F. Lambert, 2004, pp. 17-24. 
[2] S. Day, R. C. Vandervorst, T. Wanner, Topology in dynamics, differential equations, and data, Physica D: Nonlinear Phenomena 334 (2016) 1-3.

[3] H. Edelsbrunner, J. Harer, Persistent homology-a survey, Contemporary Mathematics 453 (2008) 257-282.

[4] R. Ghrist, Barcodes: the persistent topology of data, Bulletin of the American Mathematical Society 45 (2008) 61-75.

[5] G. Carlsson, Topological pattern recognition for point cloud data, Acta Numerica 23 (2014) 289-368.

[6] M. Gameiro, K. Mischaikow, W. Kalies, Topological characterization of spatial-temporal chaos, Physical Review E 70 (2004) 035203.

[7] M. Gameiro, K. Mischaikow, T. Wanner, Evolution of pattern complexity in the Cahn-Hilliard theory of phase separation, Acta Materialia 53 (2005) 693-704.

[8] K. Krishan, H. Kurtuldu, M. F. Schatz, M. Gameiro, K. Mischaikow, S. Madruga, Homology and symmetry breaking in Rayleigh-Bénard convection: Experiments and simulations, Physics of Fluids 19 (2007) 117105.

[9] H. Kurtuldu, K. Mischaikow, M. F. Schatz, Extensive scaling from computational homology and Karhunen-Loève decomposition analysis of RayleighBénard convection experiments, Physical Review Letters 107 (2011) 034503.

[10] H. Kurtuldu, K. Mischaikow, M. Schatz, Measuring the departures from the Boussinesq approximation in Rayleigh-Bénard convection experiments, Journal of Fluid Mechanics 682 (2011) 543-557.

[11] M. Kramár, A. Goullet, L. Kondic, K. Mischaikow, Quantifying force networks in particulate systems, Physica D: Nonlinear Phenomena 283 (2014) $37-55$.

[12] M. Kramár, R. Levanger, J. Tithof, B. Suri, M. Xu, M. Paul, M. F. Schatz, K. Mischaikow, Analysis of Kolmogorov flow and Rayleigh-Bénard convection using persistent homology, Physica D: Nonlinear Phenomena 334 (2016) 82-98. 
[13] C. M. Pereira, R. F. de Mello, Persistent homology for time series and spatial data clustering, Expert Systems with Applications 42 (2015) 6026-6038.

[14] J. Garland, E. Bradley, J. D. Meiss, Exploring the topology of dynamical reconstructions, Physica D: Nonlinear Phenomena 334 (2016) 49-59.

[15] S. Maletić, Y. Zhao, M. Rajković, Persistent topological features of dynamical systems, Chaos: An Interdisciplinary Journal of Nonlinear Science 26 (2016) 053105.

[16] M. Muldoon, R. MacKay, J. Huke, D. Broomhead, Topology from time series, Physica D: Nonlinear Phenomena 65 (1993) 1-16.

[17] L. C. Kinsey, Topology of surfaces. Undergraduate Texts in Mathematics, Springer, New York, 1993.

[18] R. Gilmore, M. Lefranc, The topology of chaos: Alice in stretch and squeeze land, Hoboken: Wiley \& Sons inc (2002) 518.

[19] T. Sauer, J. A. Yorke, M. Casdagli, Embedology, Journal of Statistical Physics 65 (1991) 579-616.

[20] A. Tausz, M. Vejdemo-Johansson, H. Adams, Javaplex: A research software package for persistent (co) homology, Software available at http://code. google. com/javaplex 2 (2011).

[21] C. Maria, J.-D. Boissonnat, M. Glisse, M. Yvinec, The Gudhi library: Simplicial complexes and persistent homology, in: International Congress on Mathematical Software, Springer, pp. 167-174.

[22] U. Bauer, Ripser: Efficient computation of Vietoris-Rips persistence barcodes, arXiv preprint arXiv:1908.02518 (2019).

[23] D. Sciamarella, G. Mindlin, Topological structure of chaotic flows from human speech data, Physical Review Letters 82 (1999) 1450.

[24] D. Sciamarella, G. Mindlin, Unveiling the topological structure of chaotic flows from data, Physical Review E 64 (2001) 036209.

[25] S. Barannikov, The framed Morse complex and its invariants, Adv. in Soviet Math. 21 (1994) 93-115. 
[26] M. R. Allshouse, T. Peacock, Lagrangian based methods for coherent structure detection, Chaos: An Interdisciplinary Journal of Nonlinear Science 25 (2015) 097617.

[27] G. Haller, Lagrangian coherent structures, Annual Review of Fluid Mechanics 47 (2015) 137-162.

[28] M. R. Allshouse, J.-L. Thiffeault, Detecting coherent structures using braids, Physica D: Nonlinear Phenomena 241 (2012) 95-105.

[29] K. L. Schlueter-Kuck, J. O. Dabiri, Identification of individual coherent sets associated with flow trajectories using coherent structure coloring, Chaos: An Interdisciplinary Journal of Nonlinear Science 27 (2017) 091101.

[30] M. Ghil, A century of nonlinearity in the geosciences, Earth and Space Science (2019) 1007-1042.

[31] P. Miron, F. J. Beron-Vera, M. J. Olascoaga, J. Sheinbaum, P. Pérez-Brunius, G. Froyland, Lagrangian dynamical geography of the Gulf of Mexico, Scientific Reports 7 (2017) 7021.

[32] G. Froyland, O. Junge, On fast computation of finite-time coherent sets using radial basis functions, Chaos: An Interdisciplinary Journal of Nonlinear Science 25 (2015) 087409.

[33] S. Balasuriya, Barriers and transport in unsteady flows: A Melnikov approach, volume 21 of Series on Mathematical Modeling and Computation, SIAM Press, Philadelphia, 2016.

[34] A. Hadjighasem, M. Farazmand, D. Blazevski, G. Froyland, G. Haller, A critical comparison of Lagrangian methods for coherent structure detection, Chaos: An Interdisciplinary Journal of Nonlinear Science 27 (2017) 053104.

[35] D. H. Kelley, M. R. Allshouse, N. T. Ouellette, Lagrangian coherent structures separate dynamically distinct regions in fluid flows, Physical Review E 88 (2013) 013017.

[36] R. Banisch, P. Koltai, Understanding the geometry of transport: Diffusion maps for Lagrangian trajectory data unravel coherent sets, Chaos: An Interdisciplinary Journal of Nonlinear Science 27 (2017) 035804. 
[37] J. Birman, R. F. Williams, Knotted periodic orbits in dynamical systems i: Lorenz's equations, Topology 22 (1983) 47-82.

[38] R. W. Ghrist, Branched two-manifolds supporting all links, Topology 36 (1997) 423-448.

[39] R. Gilmore, Topological analysis of chaotic dynamical systems, Reviews of Modern Physics 70 (1998) 1455.

[40] C. Letellier, J. Maquet, L. Le Sceller, G. Gouesbet, L. Aguirre, On the nonequivalence of observables in phase-space reconstructions from recorded time series, Journal of Physics A: Mathematical and General 31 (1998) 7913.

[41] C. Letellier, L. A. Aguirre, Investigating nonlinear dynamics from time series: the influence of symmetries and the choice of observables, Chaos: An Interdisciplinary Journal of Nonlinear Science 12 (2002) 549-558.

[42] C. Letellier, L. A. Aguirre, J. Maquet, Relation between observability and differential embeddings for nonlinear dynamics, Physical Review E 71 (2005) 066213.

[43] G. D. Charó, D. Sciamarella, S. Mangiarotti, G. Artana, C. Letellier, Observability of laminar bidimensional fluid flows seen as autonomous chaotic systems, Chaos: An Interdisciplinary Journal of Nonlinear Science 29 (2019) 123126.

[44] C. Letellier, L. A. Aguirre, U. Freitas, Frequently asked questions about global modeling, Chaos: An Interdisciplinary Journal of Nonlinear Science 19 (2009) 023103.

[45] S. Mangiarotti, F. Le Jean, M. Huc, C. Letellier, Global modeling of aggregated and associated chaotic dynamics, Chaos, Solitons \& Fractals 83 (2016) 82-96.

[46] F. Takens, Detecting strange attractors in turbulence, in: Dynamical systems and turbulence, Warwick 1980, Springer, 1981, pp. 366-381.

[47] A. M. Fraser, H. L. Swinney, Independent coordinates for strange attractors from mutual information, Physical Review A 33 (1986) 1134. 
[48] M. B. Kennel, R. Brown, H. D. I. Abarbanel, Determining embedding dimension for phase-space reconstruction using a geometrical construction, Physical Review A 45 (1992) 3403.

[49] H. D. Abarbanel, R. Brown, J. J. Sidorowich, L. S. Tsimring, The analysis of observed chaotic data in physical systems, Reviews of Modern Physics 65 (1993) 1331.

[50] E. Bradley, H. Kantz, Nonlinear time-series analysis revisited, Chaos: An Interdisciplinary Journal of Nonlinear Science 25 (2015) 097610.

[51] D. Broomhead, R. Jones, G. P. King, Topological dimension and local coordinates from time series data, Journal of Physics A: Mathematical and General 20 (1987) L563.

[52] S. C. Shadden, F. Lekien, J. E. Marsden, Definition and properties of Lagrangian coherent structures from finite-time Lyapunov exponents in twodimensional aperiodic flows, Physica D: Nonlinear Phenomena 212 (2005) 271-304.

[53] I. Rypina, M. G. Brown, F. J. Beron-Vera, H. Koçak, M. J. Olascoaga, I. Udovydchenkov, On the Lagrangian dynamics of atmospheric zonal jets and the permeability of the stratospheric polar vortex, Journal of the Atmospheric Sciences 64 (2007) 3595-3610.

[54] I. I. Rypina, L. J. Pratt, Trajectory encounter volume as a diagnostic of mixing potential in fluid flows, Nonlinear Processes in Geophysics 24 (2017) 189-202.

[55] P. Roca, A. Cammilleri, T. Duriez, L. Mathelin, G. Artana, Streakline-based closed-loop control of a bluff body flow, Physics of Fluids 26 (2014) 047102.

[56] S. Balasuriya, Transport between two fluids across their mutual flow interface: The streakline approach, SIAM Journal on Applied Dynamical Systems 16 (2017) 1015-1044.

[57] G. You, S. Leung, An Eulerian method for computing the coherent ergodic partition of continuous dynamical systems, Journal of Computational Physics 264 (2014) 112-132. 
[58] M. Rosalie, Templates and subtemplates of Rössler attractors from a bifurcation diagram, Journal of Physics A: Mathematical and Theoretical 49 (2016) 315101.

[59] K. L. Schlueter-Kuck, J. O. Dabiri, Coherent structure colouring: identification of coherent structures from sparse data using graph theory, Journal of Fluid Mechanics 811 (2017) 468-486.

[60] A. Hadjighasem, D. Karrasch, H. Teramoto, G. Haller, Spectral-clustering approach to Lagrangian vortex detection, Physical Review E 93 (2016) 063107. 